\title{
SUBMANIFOLDS WITH PARALLEL MEAN CURVATURE VECTOR IN PINCHED RIEMANNIAN MANIFOLDS
}

\author{
F. Fontenele
}

In this paper, we prove a generalized integral inequality for submanifolds with parallel mean curvature vector in an arbitrary Riemannian manifold, and from which we obtain a pinching theorem for compact oriented submanifolds with parallel mean curvature vector in a complete simply connected pinched Riemannian manifold, which generalizes the results obtained by Alencar-do Carmo and Hong-Wei Xu.

\section{Introduction.}

Let $M^{n}$ be an $n$-dimensional oriented closed minimal submanifold in an $(n+p)$-dimensional Riemannian manifold $N^{n+p}$. Denote by $a(x)$ and $b(x)$ the infimum and the supremum, respectively, of the sectional curvatures of $N$ at a point $x$. In [11], Hong-Wei Xu obtained the following inequality

$$
\int_{M}\left\{n b S-\left(1+\frac{1}{2} \operatorname{sgn}(p-1)\right) S^{2}-D(n, p)(b-a) S-E(n, p)(b-a)^{2}\right\} \leq 0,
$$

where $S$ is the square norm of the second fundamental form of the immersion, $\operatorname{sgn}(\cdot)$ is the standard sign function and

$$
\begin{aligned}
& D(n, p)=n+\frac{2}{3}(p-1)(n-1)^{\frac{1}{2}}, \\
& E(n, p)=\frac{1}{72} p n(n-1)(26 n-25) .
\end{aligned}
$$

Using (1.1), Xu proved in [11] the following result:

Theorem 1.3. Let $M^{n}$ be an $n$-dimensional oriented closed minimal submanifold in a complete simply connected manifold $N^{n+p}$ with $\delta(n, p) \leq K_{N} \leq$ 1, where

$$
\delta(n, p)=1-\frac{n-\frac{n}{3} \operatorname{sgn}(p-1)}{D(n, p)+2 E^{\frac{1}{2}}(n, p)}
$$




$$
E^{\frac{1}{2}}(1-c) \leq S \leq n-\frac{n}{3} \operatorname{sgn}(p-1)-\left(D+E^{\frac{1}{2}}\right)(1-c),
$$

where $c$ is the infimum of the sectional curvatures of $N^{n+p}$, then $N^{n+p}=$ $S_{1}^{n+p}$ and either $M^{n}$ is the unit sphere $S_{1}^{n}$, one of the Clifford minimal hypersurfaces $S^{k}\left(\sqrt{\frac{k}{n}}\right) \times S^{n-k}\left(\sqrt{\frac{n-k}{n}}\right), k=1,2, \ldots, n-1$, in $S_{1}^{n+1}$, or the Veronese surface in $S_{1}^{4}$.

We want to extend the above result to constant mean curvature. When dealing with submanifolds of constant mean curvature, it is convenient to replace the second fundamental form by a tensor $\phi: T_{p} M \times T_{p} M \rightarrow T_{p} M^{\perp}$ defined as follows: choose an orthonormal frame $\left\{e_{n+1}, \ldots, e_{n+p}\right\}$ of $T_{p} M^{\perp}$, and for each $n+1 \leq \alpha \leq n+p$, define maps $\phi_{\alpha}: T_{p} M \rightarrow T_{p} M$ by

$$
\phi_{\alpha}(X)=\left\langle h, e_{\alpha}\right\rangle X-A_{\alpha}(X),
$$

where $h$ is the mean curvature vector. The tensor $\phi$ is given by

$$
\phi(X, Y)=\sum_{\alpha=n+1}^{n+p}\left\langle\phi_{\alpha}(X), Y\right\rangle e_{\alpha} .
$$

The norm $|\phi|$ of $\phi$ is defined by

$$
|\phi|^{2}=\sum_{\alpha=n+1}^{n+p} \operatorname{tr} \phi_{\alpha}^{2}
$$

It is easy to see that both $\phi$ and $|\phi|$ do not depend on the choice of $\left\{e_{\alpha}\right\}$ and that

$$
|\phi|^{2}=|A|^{2}-n H^{2}
$$

where $H=|h|$. Furthermore, $|\phi| \equiv 0$ if and only if the immersion is totally umbilic.

According to Alencar-do Carmo [2], many theorems on minimal submanifolds have a natural extension to constant mean curvature if one replaces $|A|^{2}$ by $|\phi|^{2}$. This turns out to be the case in the present situation.

For each $0<r<1$, let $S^{n-1}(r) \hookrightarrow \mathbf{R}^{n}$ and $S^{1}\left(\sqrt{1-r^{2}}\right) \hookrightarrow \mathbf{R}^{2}$ be the cannonical immersions. Following [1], we call an $H(r)$-torus in $S_{1}^{n+1}$ the product immersion $S^{n-1}(r) \times S^{1}\left(\sqrt{1-r^{2}}\right) \rightarrow \mathbf{R}^{n} \times \mathbf{R}^{2}$.

In [1], H. Alencar and M. do Carmo obtained the following integral inequality for constant mean curvature immersions $f: M^{n} \rightarrow S_{1}^{n+1}$ of a compact $n$-dimensional manifold $M^{n}$ into the unit sphere $S_{1}^{n+1}$ :

$$
0 \geq \int_{M}|\nabla \phi|^{2}+\int_{M}|\phi|^{2}\left\{-|\phi|^{2}-\frac{n(n-2)}{\sqrt{n(n-1)}} H|\phi|+n\left(H^{2}+1\right)\right\} .
$$


For each $H \geq 0$, denote by $B_{H}$ the square of the positive root of $P_{H}(x)=0$, where

$$
P_{H}(x)=x^{2}+\frac{n(n-2)}{\sqrt{n(n-1)}} H x-n\left(H^{2}+1\right) .
$$

Then, from (1.10), they obtained the following theorem:

Theorem 1.12. Let $M^{n}$ be a compact and oriented manifold and let $f: M^{n} \rightarrow S_{1}^{n+1}$ have constant mean curvature $H$. If $|\phi|^{2} \leq B_{H}$ on $M$, then:

(i) Either $|\phi|^{2} \equiv 0$ (and $M^{n}$ is totally umbilic) or $|\phi|^{2} \equiv B_{H}$.

(ii) $|\phi|^{2} \equiv B_{H}$ if and only if:

(a) $H=0$ and $M^{n}$ is a Clifford torus in $S_{1}^{n+1}$;

(b) $H \neq 0, n \geq 3$, and $M^{n}$ is an $H(r)$-torus with $r^{2}<\frac{n-1}{n}$;

(c) $H \neq 0, n=2$, and $M^{n}$ is an $H(r)$-torus with $r^{2} \neq \frac{1}{2}$.

For submanifolds with parallel mean curvature vector in spheres, Walcy Santos extended the above theorem for higher codimensions [10]. Note that in the codimension one case, the mean curvature vector $h$ is parallel if and only if $H=|h|$ is constant.

For submanifolds with parallel mean curvature vector in arbitrary Riemannian manifolds, we obtain here the following integral inequality, which generalizes the inequalities obtained by other authors $([\mathbf{1}, \mathbf{1 0}, \mathbf{1 1}]$, etc.):

Theorem 1.13. Let $M^{n}$ be a compact and orientable manifold and let $f: M^{n} \rightarrow N^{n+p}$ be an immersion with parallel mean curvature vector $h$ in an $(n+p)$-dimensional Riemannian manifold $N^{n+p}$. Denote by $a(x)$ and $b(x)$ the infimum and the supremum, respectively, of the sectional curvatures of $N$ at a point $x$. Then

$$
\begin{aligned}
0 \geq \int_{M}\{ & -E(b-a)^{2}-\frac{2}{3} n(n-1)^{\frac{1}{2}}(p-1)(b-a) H^{2} \\
& +\left(n a+n H^{2}-\frac{2}{3}(n-1)^{\frac{1}{2}}(p-1)(b-a)\right)|\phi|^{2} \\
& \left.-\frac{n(n-2)}{\sqrt{n(n-1)}}\left|\phi_{h}\right||\phi|^{2}-\theta_{p, h}|\phi|^{4}\right\}
\end{aligned}
$$

where $H=|h|$ and

$$
\theta_{p, h}= \begin{cases}1+\frac{1}{2} \operatorname{sgn}(p-1), & \text { if } p=1 \quad \text { or } \quad h=0 \\ 1+\frac{1}{2} \operatorname{sgn}(p-2), & \text { otherwise. }\end{cases}
$$


When $H=0$, inequality (1.14) becomes the inequality (1.1) obtained by Hong-Wei Xu [11], and in the case $p=1$ and $N^{n+1}=S_{1}^{n+1}$, we reobtain (up to the term $\int_{M}|\nabla \phi|^{2}$ ) inequality (1.10) obtained by Alencar-do Carmo [1]. Furthermore, if $p \geq 2, N^{n+p}=S_{1}^{n+p}$ and $H \neq 0$, inequality (1.14) becomes

$$
0 \geq \int_{M}|\phi|^{2}\left\{n\left(1+H^{2}\right)-\frac{n(n-2)}{\sqrt{n(n-1)}}\left|\phi_{h}\right|-\left(1+\frac{1}{2} \operatorname{sgn}(p-2)\right)|\phi|^{2}\right\}
$$

which is stronger than the inequality obtained by Santos ([10], inequality $(2.14))$.

From (1.14) and the obvious inequality

$$
\frac{n(n-2)}{\sqrt{n(n-1)}}\left|\phi_{h}\right||\phi|^{2} \leq \frac{n(n-2)}{\sqrt{n(n-1)}} H|\phi|^{3},
$$

we obtain

$$
\begin{aligned}
0 \geq \int_{M}\{ & -E(b-a)^{2}-\frac{2}{3} n(n-1)^{\frac{1}{2}}(p-1)(b-a) H^{2} \\
& +\left(n a+n H^{2}-\frac{2}{3}(n-1)^{\frac{1}{2}}(p-1)(b-a)\right)|\phi|^{2} \\
& \left.-\frac{n(n-2)}{\sqrt{n(n-1)}} H|\phi|^{3}-\theta_{p, h}|\phi|^{4}\right\}
\end{aligned}
$$

In order to generalize Theorems (1.3) and (1.12), we need some notation. We set

$$
\begin{gathered}
F(n, p, H)=\left[\left(1+\theta_{p, h}\right) E^{\frac{1}{2}}+D\right] E^{\frac{1}{2}}, \\
G(n, p, H)=n\left(1+H^{2}\right) E^{\frac{1}{2}}-\frac{2}{3} n(n-1)^{\frac{1}{2}}(p-1) H^{2}, \\
\lambda(n, p, H)=\frac{-\frac{n(n-2)}{\sqrt{n(n-1)}} H E^{\frac{3}{4}}+\sqrt{\frac{n(n-2)^{2}}{n-1} H^{2} E^{\frac{3}{2}}+4 F G}}{2 F},
\end{gathered}
$$

and for $p \geq 1$ and any real numbers $H \geq 0, c \leq 1$, we define a polynomial 
$P_{H, c, p}$ by

$$
\begin{aligned}
P_{H, c, p}(x)= & -E(1-c)^{2}-\frac{2}{3} n(n-1)^{\frac{1}{2}}(p-1)(1-c) H^{2} \\
& +\left(n c+n H^{2}-\frac{2}{3}(n-1)^{\frac{1}{2}}(p-1)(1-c)\right) x^{2} \\
& -\frac{n(n-2)}{\sqrt{n(n-1)}} H x^{3}-\theta_{p, h} x^{4} .
\end{aligned}
$$

By observing the sign of the coefficients of the above polynomial, it follows from the Descartes' rule of sign (see [9], page 60, Corollary 35) that the equation $P_{H, c, p}(x)=0$ has at most two positive real roots, that we denote, if they exist, by $x_{1}(c) \leq x_{2}(c)$.

In what follows, we denote by $S_{\tilde{c}}^{n} \rightarrow \longrightarrow S_{c}^{n+p}$ the umbilical immersion of $S_{\tilde{c}}^{n}$ in $S_{c}^{n+p}$.

Using (1.18), we can finally establish our main result:

Theorem 1.23. Let $M^{n}$ be a compact and oriented manifold and let $f: M^{n} \rightarrow$ $N^{n+p}$ be an immersion in a complete and simply connected manifold $N^{n+p}$. Suppose the mean curvature vector $h$ is parallel in the normal connection and that $G(n, p, H)>0$, where $H=|h|$. If

$$
1-\lambda^{2}(n, p, H) \leq K_{N} \leq 1
$$

and

$$
x_{1}(c) \leq|\phi| \leq x_{2}(c)
$$

then $N^{n+p}=S_{1}^{n+p}$ and either $|\phi|^{2} \equiv 0$ (and the immersion is totally umbilic) or $|\phi|^{2} \equiv B_{H, p}$, where $B_{H, p}$ is the square of the positive root of $P_{H, 1, p}(x)=0$. Furthermore, $|\phi|^{2} \equiv B_{H, p}$ if and only if:

(a) $p=1, H=0$ and $M^{n}$ is a Clifford torus $S^{m}\left(\sqrt{\frac{m}{n}}\right) \times S^{n-m}\left(\sqrt{\frac{n-m}{n}}\right) \subset$ $S_{1}^{n+1}$

(b) $p=1, H>0$ and $M^{n}$ is an $H(r)$-torus $S^{n-1}(r) \times S^{1}\left(r_{1}\right) \subset S_{1}^{n+1}$, where $r^{2}+r_{1}^{2}=1$. If $n \geq 3$ we have $r^{2}<\frac{n-1}{n}$, and if $n=2$ we have $r^{2} \neq \frac{1}{2}$

(c) $p=2, n=2$ and $M^{n}$ is a Clifford torus

$$
S^{1}\left(\sqrt{\frac{1}{2\left(1+H^{2}\right)}}\right) \times S^{1}\left(\sqrt{\frac{1}{2\left(1+H^{2}\right)}}\right) \subset S_{1+H^{2}}^{3} \underset{u}{\longrightarrow} \longrightarrow S_{1}^{4} ;
$$


(d) $p=2, n=2, H>0$ and for each $H_{o}, 0<H_{o}<H, M^{n}$ is an $H_{1}(r)$-torus $S^{1}(r) \times S^{1}\left(r_{1}\right) \subset S_{1+H_{o}^{2}}^{3} \rightarrow \longrightarrow S_{1}^{4}$, where $H_{o}^{2}+H_{1}^{2}=H^{2}$, $r^{2}+r_{1}^{2}=\left(1+H_{o}^{2}\right)^{-1}$ and $r^{2} \neq \frac{1}{2}\left(1+H_{o}^{2}\right)^{-1} ;$

(e) $p=3, n=2$ and $M$ is the Veronese surface $M^{2} \subset S_{1+H^{2}}^{4} \underset{u}{\longrightarrow} \longrightarrow S_{1}^{5}$.

Remark 1.26. In [10], W. Santos classified the compact orientable submanifolds of $S_{1}^{n+p}$ with parallel mean curvature vector and for which

$$
|\phi|^{2} \leq \bar{\theta}_{p, h}\left\{n\left(1+H^{2}\right)-\frac{n(n-2)}{\sqrt{n(n-1)}}\left|\phi_{h}\right|\right\},
$$

where

$$
\bar{\theta}_{p, h}= \begin{cases}1 /(2-1 / p), & \text { if } p=1 \quad \text { and } h=0 \\ 1 /(2-1 /(p-1)), & \text { otherwise }\end{cases}
$$

She obtained more examples than those of Theorem (1.23). The reason is that, when $N^{n+p}=S_{1}^{n+p}$, condition (1.25) becomes $|\phi|^{2} \leq B_{H, p}$, which is easily seen to be stronger (for $p \leq 3$ ) than (1.27) (see (4.14) below). The case of equality in Theorem (1.23) was inspired in the proof of the theorem of Santos [10].

Remark 1.28. Condition $G(n, p, H)>0$ in Theorem (1.23) is technical and is probably needless. It should be noticed that $G(n, p, H)>0$ if $p \leq$ $\max \{3, n\}$ or if $H=0$ (for all examples appearing in Theorem (1.23), we have $p \leq 3)$.

Remark 1.29. When $p=1$ and $N^{n+1}=S_{1}^{n+1}$, condition (1.24) is immediately satisfied and (1.25) becomes the hypothesis of Theorem (1.12). In the case $H=0$, it is easy to see that $1-\lambda^{2}(n, p, 0) \leq \delta(n, p)$ and so the condition (1.24) is weaker than the hypothesis $\delta(n, p) \leq K_{N} \leq 1$ in Theorem (1.3). Furthermore, for each $c \geq \delta(n, p)$, it is possible to prove that

$$
x_{1} \leq E^{\frac{1}{2}}(1-c) \leq n-\frac{n}{3} \operatorname{sgn}(p-1)-\left(D+E^{\frac{1}{2}}\right)(1-c) \leq x_{2},
$$

and thus (1.25) is weaker than (1.5). Therefore, if $p=1$ Theorem (1.23) extends Theorem (1.12), and if $H=0$ Theorem (1.23) is stronger than Theorem (1.3).

Remark 1.30. Note that for both Theorems (1.3) and (1.23) to make sense, it is necessary to assure the existence of two positive real roots of $P_{H, c, p}(x)=0$. In the case $H=0, P_{H, c, p}$ is a quadratic polynomial in $S$, and we have only to force its discriminant to be nonnegative. If $H \neq 0$, however, $P_{H, c, p}$ is a quartic polynomial in $|\phi|$ and we overcome this difficulty in a different way (see the proof of Theorem (1.23)). 
Condition (1.25) in Theorem (1.23) can be replaced by $\xi_{1}(c) \leq|\phi|^{2} \leq$ $\xi_{2}(c)$, where $\xi_{1}(c)$ and $\xi_{2}(c)$ satisfy

$$
\begin{aligned}
& x_{1}^{2} \leq \xi_{1}(c) \leq \xi_{2}(c) \leq x_{2}^{2}, \\
& \xi_{1}(1)=0, \quad \xi_{2}(1)=B_{H, p} .
\end{aligned}
$$

For example, if $p=1$ and, if instead of (1.24), we require

$$
1-\frac{B_{H}}{n+2 E^{1 / 2}} \leq K_{N} \leq 1
$$

we can take $\xi_{1}(c)=E^{1 / 2}(1-c)$ and $\xi_{2}(c)=B_{H}-\left(n+E^{1 / 2}\right)(1-c)$.

More precisely, we have the following result:

Theorem 1.32. Let $f: M^{n} \rightarrow N^{n+1}$ be a constant mean curvature immersion of a compact oriented manifold $M^{n}$ into a complete simply connected manifold $N^{n+1}$ satisfying (1.31). If

$$
E^{\frac{1}{2}}(1-c) \leq|\phi|^{2} \leq B_{H}-\left(n+E^{\frac{1}{2}}\right)(1-c),
$$

then $N^{n+1}=S_{1}^{n+1}$ and either $|\phi|^{2} \equiv 0$ (and $M^{n}$ is totally umbilic) or $|\phi|^{2} \equiv$ $B_{H}$. We have $|\phi|^{2} \equiv B_{H}$ if and only if (a) or (b) in Theorem (1.23) occurs.

The work is organized as follows: in section 2 we obtain a formula for the Laplacian of the second fundamental form of an immersion $M^{n} \hookrightarrow N^{n+p}$ in a general framework: no assumption on the codimension, or on the mean curvature vector or on the ambient space is made. In section 3 we consider the case of parallel mean curvature vector, and after some estimates, we prove Theorem (1.13). In section 4 we prove Theorems (1.23) and (1.32).

Acknowledgements. This work is the author's doctoral thesis at Instituto de Matemática Pura e Aplicada. The author wants to thank Manfredo Perdigão do Carmo for his orientation and Walcy Santos and Sergio Luiz Silva for helpful conversations and suggestions.

\section{The Laplacian of the Second Fundamental Form.}

In this section we shall compute the Laplacian of the second fundamental form of an immersion $f: M^{n} \rightarrow N^{n+p}$ by using moving frames. Notations will be as in [4]. We shall make use of the following convention on the ranges of indices:

$$
\begin{gathered}
1 \leq A, B, C, \ldots, \leq n+p ; \quad 1 \leq i, j, k, \ldots, \leq n \\
n+1 \leq \alpha, \beta, \gamma, \ldots, \leq n+p .
\end{gathered}
$$


Choose a local field of orthonormal frames $e_{1}, \ldots, e_{n+p}$ in $N$ such that, restricted to $M$, the vectors $e_{1}, \ldots, e_{n}$ are tangent to $M$. Let $\left\{w_{A}\right\}$ and $\left\{w_{A B}\right\}$ be the fields of dual frames and the connection 1-forms of $N$, respectively. Restricting the forms to $M$, we have

$$
w_{\alpha i}=\sum_{j} h_{i j}^{\alpha} w_{j}, \quad h_{i j}^{\alpha}=h_{j i}^{\alpha},
$$

where the $h_{i j}^{\alpha}$ are the coefficients of the second fundamental form of the immersion. The equations of Gauss and Ricci are:

$$
\begin{aligned}
& R_{j k \ell}^{i}=K_{j k \ell}^{i}+\sum_{\alpha}\left(h_{i k}^{\alpha} h_{j \ell}^{\alpha}-h_{i \ell}^{\alpha} h_{j k}^{\alpha}\right), \\
& R_{\beta k \ell}^{\alpha}=K_{\beta k \ell}^{\alpha}+\sum_{i}\left(h_{i k}^{\alpha} h_{i \ell}^{\beta}-h_{i \ell}^{\alpha} h_{i k}^{\beta}\right),
\end{aligned}
$$

where $R_{\beta k \ell}^{\alpha}, R_{j k \ell}^{i}$ and $K_{B C D}^{A}$ are the normal curvature tensor, the curvature tensor of $M$ and the curvature tensor of $N$, respectively. We define the covariant derivatives of $h_{i j}^{\alpha}$ by

$$
\begin{gathered}
\sum_{k} h_{i j k}^{\alpha} w_{k}=d h_{i j}^{\alpha}+\sum_{s} h_{s j}^{\alpha} w_{i s}+\sum_{s} h_{i s}^{\alpha} w_{j s}+\sum_{\beta} h_{i j}^{\beta} w_{\alpha \beta} \\
\sum_{\ell} h_{i j k \ell}^{\alpha} w_{\ell}=d h_{i j k}^{\alpha}+\sum_{s} h_{s j k}^{\alpha} w_{i s}+\sum_{s} h_{i s k}^{\alpha} w_{j s}+\sum_{s} h_{i j s}^{\alpha} w_{s}+\sum_{\beta} h_{i j k}^{\beta} w_{\alpha \beta} .
\end{gathered}
$$

We have

$$
\begin{gathered}
h_{i j k}^{\alpha}-h_{i k j}^{\alpha}=-K_{i j k}^{\alpha}, \\
h_{i j k \ell}^{\alpha}-h_{i j \ell k}^{\alpha}=\sum_{s} h_{s j}^{\alpha} R_{i k \ell}^{s}+\sum_{s} h_{i s}^{\alpha} R_{j k \ell}^{s}+\sum_{\beta} h_{i j}^{\beta} R_{\beta k \ell}^{\alpha} .
\end{gathered}
$$

We define the Laplacian of the second fundamental form by $\Delta h_{i j}^{\alpha}=$ $\sum_{k} h_{i j k k}^{\alpha}$. Using (2.4) and (2.5) we obtain

$$
\begin{aligned}
\Delta h_{i j}^{\alpha}=\sum_{k}\left(h_{k k i j}^{\alpha}\right. & \left.-K_{k i k j}^{\alpha}-K_{i j k k}^{\alpha}\right)+\sum_{k, m} h_{k m}^{\alpha} R_{i j k}^{m} \\
& +\sum_{k, m} h_{m i}^{\alpha} R_{k j k}^{m}-\sum_{k, \beta} h_{k i}^{\beta} R_{\beta j k}^{\alpha} .
\end{aligned}
$$

Substituting (2.2) and (2.3) into the above formula, we have

$$
\begin{aligned}
\Delta h_{i j}^{\alpha}= & \sum_{k}\left(h_{k k i j}^{\alpha}-K_{k i k j}^{\alpha}-K_{i j k k}^{\alpha}\right)+\sum_{k, m}\left(h_{k m}^{\alpha} K_{i j k}^{m}+h_{m i}^{\alpha} K_{k j k}^{m}\right) \\
+ & \sum_{k, \beta} h_{k i}^{\beta} K_{\beta k j}^{\alpha}+\sum_{m, k, \beta}\left(h_{m i}^{\alpha} h_{m j}^{\beta} h_{k k}^{\beta}+2 h_{k m}^{\alpha} h_{m j}^{\beta} h_{i k}^{\beta}\right. \\
& \left.-h_{k m}^{\alpha} h_{k m}^{\beta} h_{i j}^{\beta}-h_{m i}^{\alpha} h_{k m}^{\beta} h_{k j}^{\beta}-h_{m j}^{\alpha} h_{k i}^{\beta} h_{k m}^{\beta}\right),
\end{aligned}
$$


and then

$$
\begin{aligned}
\frac{1}{2} \Delta|A|^{2} & =\sum_{i, j, k, \alpha}\left(h_{i j k}^{\alpha}\right)^{2}+\sum_{i, j, \alpha} h_{i j}^{\alpha} \Delta h_{i j}^{\alpha} \\
& =\sum_{i, j, k, \alpha}\left(h_{i j k}^{\alpha}\right)^{2}+\sum_{i, j, k, \alpha}\left(h_{i j}^{\alpha} h_{k k i j}^{\alpha}-h_{i j}^{\alpha} K_{k i k j}^{\alpha}-h_{i j}^{\alpha} K_{i j k k}^{\alpha}\right) \\
& +\sum_{i, j, k, m, \alpha}\left(h_{i j}^{\alpha} h_{m j}^{\alpha} K_{k i k}^{m}+h_{i j}^{\alpha} h_{m k}^{\alpha} K_{i j k}^{m}\right)+\sum_{i, j, k, \alpha, \beta} h_{i j}^{\alpha} h_{k i}^{\beta} K_{\beta k j}^{\alpha} \\
& -\sum_{i, j, k, \ell, \alpha, \beta} h_{i j}^{\alpha} h_{k \ell}^{\alpha} h_{i j}^{\beta} h_{k \ell}^{\beta}+\sum_{i, j, k, \ell, \alpha, \beta} h_{i j}^{\alpha} h_{i \ell}^{\alpha} h_{j \ell}^{\beta} h_{k k}^{\beta} \\
& -\sum_{i, j, k, \ell, \alpha, \beta}\left(h_{i k}^{\alpha} h_{j k}^{\beta}-h_{j k}^{\alpha} h_{i k}^{\beta}\right)\left(h_{i \ell}^{\alpha} h_{j \ell}^{\beta}-h_{j \ell}^{\alpha} h_{i \ell}^{\beta}\right) .
\end{aligned}
$$

Remark 2.7. In the case $H \equiv 0$, the terms

$$
\sum_{i, j, k, \alpha} h_{i j}^{\alpha} h_{k k i j}^{\alpha} \quad \text { and } \sum_{i, j, k, \ell, \alpha, \beta} h_{i j}^{\alpha} h_{i \ell}^{\alpha} h_{j \ell}^{\beta} h_{k k}^{\beta}
$$

in formula (2.6) vanish, and (2.6) reduces to the corresponding formula encountered in $[\mathbf{1 1}]$.

\section{Estimates and the Proof of Theorem (1.13).}

From now on, we assume that the immersion has parallel mean curvature vector in the normal connection. In this case, we have the following lemma:

Lemma 3.1. For an immersion $M^{n} \hookrightarrow N^{n+p}$ with parallel mean curvature vector $h$, we have

$$
\nabla \phi=-\nabla \sigma
$$

where $\sigma: T_{p} M \times T_{p} M \rightarrow T_{p} M^{\perp}$ denotes the second fundamental form and $\nabla \phi$ and $\nabla \sigma$ denote the gradient of the tensors $\phi$ and $\sigma$, respectively.

Proof. From (1.7), it is immediate to verify that

$$
\phi(X, Y)=\langle X, Y\rangle h-\sigma(X, Y)
$$

for any $X, Y \in T M$. Now fix a point $p \in M$ and choose a local orthonormal frame $\left\{e_{1}, \ldots, e_{n}\right\}$ such that $\nabla_{e_{i}} e_{j}(p)=0$ for all $i, j$. Since $h$ is parallel, we then have in $p$ that

$$
\begin{aligned}
(\nabla \phi)\left(e_{i}, e_{j}, e_{k}\right) & =\left(\nabla_{e_{k}} \phi\right)\left(e_{i}, e_{j}\right)=\nabla_{e_{k}} \phi\left(e_{i}, e_{j}\right)=\nabla_{e_{k}}\left(\left\langle e_{i}, e_{j}\right\rangle h-\sigma\left(e_{i}, e_{j}\right)\right) \\
& =-\nabla_{e_{k}} \sigma\left(e_{i}, e_{j}\right)=-\left(\nabla_{e_{k}} \sigma\right)\left(e_{i}, e_{j}\right)=-\nabla \sigma\left(e_{i}, e_{j}, e_{k}\right)
\end{aligned}
$$


for all $i, j, k$, and the lemma is proved.

From the above lemma we obtain that $\nabla^{2} \phi=-\nabla^{2} \sigma$, and so $\phi_{i j k \ell}^{\alpha}=$ $-h_{i j k \ell}^{\alpha}$. Since $\operatorname{tr} \phi_{\alpha}=0$, we conclude that

$$
\sum_{i, j, k, \alpha} h_{i j}^{\alpha} h_{k k i j}^{\alpha}=-\sum_{i, j, k, \alpha}\left(\left\langle h, e_{\alpha}\right\rangle \delta_{i j}-\phi_{i j}^{\alpha}\right) \phi_{k k i j}^{\alpha}=0
$$

Using this fact, formula (2.6) becomes

$$
\begin{aligned}
\frac{1}{2} \Delta|A|^{2} & =\sum_{i, j, k, \alpha}\left(h_{i j k}^{\alpha}\right)^{2}-\sum_{i, j, k, \alpha}\left(h_{i j}^{\alpha} K_{k i k j}^{\alpha}+h_{i j}^{\alpha} K_{i j k k}^{\alpha}\right) \\
& +\sum_{i, j, k, m, \alpha}\left(h_{i j}^{\alpha} h_{m j}^{\alpha} K_{k i k}^{m}+h_{i j}^{\alpha} h_{m k}^{\alpha} K_{i j k}^{m}\right)+\sum_{i, j, k, \alpha, \beta} h_{i j}^{\alpha} h_{k i}^{\beta} K_{\beta k j}^{\alpha} \\
& -\sum_{i, j, k, \ell, \alpha, \beta} h_{i j}^{\alpha} h_{k \ell}^{\alpha} h_{i j}^{\beta} h_{k \ell}^{\beta}+\sum_{i, j, k, \ell, \alpha, \beta} h_{i j}^{\alpha} h_{i \ell}^{\alpha} h_{j \ell}^{\beta} h_{k k}^{\beta} \\
& -\sum_{i, j, k, \ell, \alpha, \beta}\left(h_{i k}^{\alpha} h_{j k}^{\beta}-h_{j k}^{\alpha} h_{i k}^{\beta}\right)\left(h_{i \ell}^{\alpha} h_{j \ell}^{\beta}-h_{j \ell}^{\alpha} h_{i \ell}^{\beta}\right) .
\end{aligned}
$$

Remark 3.3. When the ambient manifold has constant curvature, the second and the fourth terms in (3.2) vanish, and we obtain the formula given in Erbacher ([6], formula (12)).

To obtain estimates for the terms appearing in the right hand side of (3.2), we will use the following propositions. Except for the equality case in part (i), Proposition (3.4) below is proved in [7, pages 92-94].

Proposition 3.4 ([7], see also [11]). If $N$ is a Riemannian manifold and $a \leq K_{N} \leq b$ at a point $x \in N$, then, at this point,

- (i) $\left|K_{A B A C}\right| \leq \frac{1}{2}(b-a)$, for $B \neq C$;

- (ii) $\left|K_{A B C D}\right| \leq \frac{2}{3}(b-a)$, for $A, B, C, D$ distinct two by two.

Equality in (i) implies that $K_{A C}=K_{B C}$.

Proposition 3.5 [10]. Let $x_{i}, y_{i} i=1, \ldots, n$, be real numbers such that $\sum_{i} x_{i}=0=\sum_{i} y_{i}$. Then

$$
-\frac{n-2}{\sqrt{n(n-1)}} A^{2} B \leq \sum_{i} x_{i}^{2} y_{i} \leq \frac{n-2}{\sqrt{n(n-1)}} A^{2} B,
$$

where $A^{2}=\sum_{i} x_{i}^{2}$ and $B^{2}=\sum_{i} y_{i}^{2}$. 
Proposition 3.6 (see $[4,8,11])$. Let $A_{n+1}, A_{n+2}, \ldots, A_{n+p}$ be symmetric $(n \times n)$-matrices. Denote $S_{\alpha \beta}=\operatorname{tr}\left(A_{\alpha}^{t} A_{\beta}\right)$ and $N(A)=\operatorname{tr}\left(A^{t} A\right)$. Then,

$$
\sum_{\alpha, \beta} N\left(A_{\alpha} A_{\beta}-A_{\beta} A_{\alpha}\right)+\sum_{\alpha, \beta} S_{\alpha \beta}^{2} \leq\left(1+\frac{1}{2} \operatorname{sgn}(p-1)\right)\left(\sum_{\alpha} \operatorname{tr} A_{\alpha}^{2}\right)^{2} .
$$

For future use, we shall prove Proposition (3.4)(i).

Proof of Proposition (3.4)(i). Let A, B, $C$ be mutually distinct. A simple computation shows that

$$
K\left(e_{A}, \xi e_{B}+\eta e_{C}\right)=\frac{\xi^{2} K_{A B}-2 \xi \eta K_{A B A C}+\eta^{2} K_{A C}}{\xi^{2}+\eta^{2}},
$$

for any real numbers $\xi, \eta$. Since $a \leq K\left(e_{A}, \xi e_{B}+\eta e_{C}\right) \leq b$, we obtain

$$
\begin{aligned}
& \left(K_{A B}-a\right) \xi^{2}-2 \xi \eta K_{A B A C}+\left(K_{A C}-a\right) \eta^{2} \geq 0 \\
& \left(b-K_{A B}\right) \xi^{2}+2 \xi \eta K_{A B A C}+\left(b-K_{A C}\right) \eta^{2} \geq 0
\end{aligned}
$$

for all $\xi, \eta \in \mathbf{R}$, which implies that

$$
\begin{aligned}
& \left|K_{A B A C}\right| \leq \sqrt{\left(K_{A B}-a\right)\left(K_{A C}-a\right)}, \\
& \left|K_{A B A C}\right| \leq \sqrt{\left(b-K_{A B}\right)\left(b-K_{A C}\right)} .
\end{aligned}
$$

Since

$$
\sqrt{\left(K_{A B}-a\right)\left(K_{A C}-a\right)} \leq \frac{K_{A B}+K_{A C}-2 a}{2}
$$

and

$$
\sqrt{\left(b-K_{A B}\right)\left(b-K_{A C}\right)} \leq \frac{2 b-K_{A B}-K_{A C}}{2},
$$

we conclude from (3.7) and (3.8) that

$$
\left|K_{A B A C}\right| \leq \frac{b-a}{2} .
$$

Equality in (3.11) implies that all the above inequalities become equalities. In particular equality holds in (3.9), which shows that $K_{A B}=K_{A C}$.

To estimate the right hand side of (3.2), we will study each term separately. The integral inequality of the following lemma is a generalization of a corresponding inequality in Xu's paper [11], which is a key step in the proof of Theorem (1.3). 
Lemma 3.12 (see [11]).

$$
\int_{M}\left\{\sum_{i, j, k, \alpha}\left(\left(h_{i j k}^{\alpha}\right)^{2}-h_{i j}^{\alpha} K_{k i k j}^{\alpha}-h_{i j}^{\alpha} K_{i j k k}^{\alpha}\right)\right\} \geq-\int_{M} E(n, p)(b-a)^{2},
$$

where $E(n, p)$ is given by (1.2).

Proof. It is straightforward to verify that $\sum_{i, j, k, \alpha}\left(\left(h_{i j k}^{\alpha}\right)^{2}-h_{i j}^{\alpha} K_{k i k j}^{\alpha}-h_{i j}^{\alpha} K_{i j k k}^{\alpha}\right)$ is a globally defined function, and hence, the integral of the left hand side of the inequality makes sense.

Next, we claim that

$$
-\sum_{i, j, k, \alpha}\left(h_{i k}^{\alpha} K_{j i j k}^{\alpha}+h_{i j}^{\alpha} K_{i j k k}^{\alpha}\right)=\sum_{i, j, k, \alpha}\left(h_{i k k}^{\alpha} K_{j i j}^{\alpha}+h_{i j k}^{\alpha} K_{i j k}^{\alpha}\right)-\operatorname{div} V
$$

where $V$ is the globally defined vector field

$$
V=\sum_{i, j, k, \alpha}\left(h_{i k}^{\alpha} K_{j i k}^{\alpha}+h_{i j}^{\alpha} K_{i j k}^{\alpha}\right) e_{k}
$$

and $\operatorname{div} V$ means the divergence of $V$. For this, fix a point $p \in M$ and choose a local orthonormal frame $\left\{e_{1}, \ldots, e_{n}\right\}$ in $M$ such that $\nabla_{e_{i}} e_{j}(p)=0$ for all $i, j$. In $p$ we have

$$
\begin{aligned}
& -\sum_{i, j, k, \alpha}\left(h_{i k}^{\alpha} K_{j i j k}^{\alpha}+h_{i j}^{\alpha} K_{i j k k}^{\alpha}\right)=-\sum_{i, j, k, \alpha} e_{k}\left(h_{i k}^{\alpha} K_{j i j}^{\alpha}+h_{i j}^{\alpha} K_{i j k}^{\alpha}\right)+ \\
& +\sum_{i, j, k, \alpha}\left(h_{i k k}^{\alpha} K_{j i j}^{\alpha}+h_{i j k}^{\alpha} K_{i j k}^{\alpha}\right)=\sum_{i, j, k, \alpha}\left(h_{i k k}^{\alpha} K_{j i j}^{\alpha}+h_{i j k}^{\alpha} K_{i j k}^{\alpha}\right)-\operatorname{div} V,
\end{aligned}
$$

where $e_{k}(\cdot)$ denotes directional derivative, and since $p$ is arbitrary, the claim is proved.

Using (2.4), Proposition (3.4), Lemma (3.1) and the fact that $\operatorname{tr} \phi_{\alpha}=0$ for all $\alpha$, we have

$$
\begin{aligned}
\sum_{i, j, k, \alpha} h_{i k k}^{\alpha} K_{j i j}^{\alpha} & =\sum_{i, j, k, \alpha}\left(h_{k k i}^{\alpha}-K_{k i k}^{\alpha}\right) K_{j i j}^{\alpha} \\
& =-\sum_{i, j, k, \alpha}\left(\phi_{k k i}^{\alpha}+K_{k i k}^{\alpha}\right) K_{j i j}^{\alpha} \\
& =-\sum_{i, \alpha}\left(\sum_{j} K_{j i j}^{\alpha}\right)^{2} \\
& \geq-\frac{1}{4} p n(n-1)^{2}(b-a)^{2} .
\end{aligned}
$$


On the other hand, using (2.4) and Proposition (3.4), we obtain

$$
\begin{aligned}
\sum_{i, j, k, \alpha}\left(h_{i j k}^{\alpha}\right)^{2} & +\sum_{i, j, k, \alpha} h_{i j k}^{\alpha} K_{i j k}^{\alpha} \geq-\frac{1}{4} \sum_{i, j, k, \alpha}\left(K_{i j k}^{\alpha}\right)^{2} \\
& \geq-\frac{1}{4} \sum_{\alpha} \sum_{i, j, k \text { distinct }}\left(K_{i j k}^{\alpha}\right)^{2}-\frac{1}{2} \sum_{\alpha} \sum_{i \neq j}\left(K_{i j i}^{\alpha}\right)^{2} \\
& \geq-\frac{1}{9} p n(n-1)(n-2)(b-a)^{2}-\frac{1}{8} p n(n-1)(b-a)^{2} .
\end{aligned}
$$

The conclusion now follows from (3.13), (3.14) and (3.15), by using Green's divergence theorem.

Lemma 3.16 (see $[11]$ ).

$$
\begin{gathered}
\sum_{i, j, k, m, \alpha}\left(h_{i j}^{\alpha} h_{m j}^{\alpha} K_{k i k}^{m}+h_{i j}^{\alpha} h_{m k}^{\alpha} K_{i j k}^{m}\right)+\sum_{i, j, k, \alpha, \beta} h_{i j}^{\alpha} h_{k i}^{\beta} K_{\beta k j}^{\alpha} \\
\geq n a|\phi|^{2}-\frac{2}{3}(n-1)^{\frac{1}{2}}(p-1)(b-a)|A|^{2}
\end{gathered}
$$

Proof. Fix a vector $e_{\alpha}$ and let $\left\{e_{i}\right\}$ be a frame diagonalizing the matrix $\left(h_{i j}^{\alpha}\right)$ with eigenvalues $\lambda_{i}^{\alpha}, i=1, \ldots, n$. This frame also diagonalizes $\left(\phi_{i j}^{\alpha}\right)$, with eigenvalues $\mu_{i}^{\alpha}=\left\langle h, e_{\alpha}\right\rangle-\lambda_{i}^{\alpha}$. By Proposition (3.4), we have

$$
\begin{aligned}
\sum_{i, j, k, \beta} h_{i j}^{\alpha} h_{k i}^{\beta} K_{\beta k j}^{\alpha}= & \sum_{i, k, \beta} h_{k i}^{\beta} \lambda_{i}^{\alpha} K_{\beta k i}^{\alpha} \\
\geq & -\sum_{i \neq k, \beta \neq \alpha} \frac{2}{3}(b-a)\left|h_{k i}^{\beta} \lambda_{i}^{\alpha}\right| \\
\geq & -\sum_{i \neq k, \beta \neq \alpha} \frac{1}{3}(b-a)\left((n-1)^{\frac{1}{2}}\left(h_{k i}^{\beta}\right)^{2}+(n-1)^{-\frac{1}{2}}\left(\lambda_{i}^{\alpha}\right)^{2}\right) \\
\geq & -\frac{1}{3}(n-1)^{\frac{1}{2}}(b-a) \sum_{\beta \neq \alpha} \operatorname{tr} A_{\beta}^{2} \\
& -\frac{1}{3}(n-1)^{\frac{1}{2}}(p-1)(b-a) \operatorname{tr} A_{\alpha}^{2} .
\end{aligned}
$$


On the other hand, using the fact that $\operatorname{tr} \phi_{\alpha}=0$, we have

$$
\begin{aligned}
\sum_{i, j, k, m}\left(h_{i j}^{\alpha} h_{m j}^{\alpha} K_{k i k}^{m}+h_{i j}^{\alpha} h_{m k}^{\alpha} K_{i j k}^{m}\right) & =\sum_{i, k}\left(\lambda_{i}^{\alpha}\right)^{2} K_{k i k}^{i}+\sum_{i, k} \lambda_{k}^{\alpha} \lambda_{i}^{\alpha} K_{i i k}^{k} \\
& =\frac{1}{2} \sum_{i, k}\left(\lambda_{i}^{\alpha}-\lambda_{k}^{\alpha}\right)^{2} K_{k i k}^{i} \\
& =\frac{1}{2} \sum_{i, k}\left(\mu_{i}^{\alpha}-\mu_{k}^{\alpha}\right)^{2} K_{k i k}^{i} \\
& \geq \frac{1}{2} a \sum_{i, k}\left(\mu_{i}^{\alpha}-\mu_{k}^{\alpha}\right)^{2} \\
& =n a \operatorname{tr} \phi_{\alpha}^{2} .
\end{aligned}
$$

The lemma now follows from (3.17) and (3.18).

\section{Lemma 3.19.}

$$
-\sum_{i, j, k, \ell, \alpha, \beta} h_{i j}^{\alpha} h_{k \ell}^{\alpha} h_{i j}^{\beta} h_{k \ell}^{\beta}=-\sum_{\alpha, \beta}\left(\operatorname{tr} \phi_{\alpha} \phi_{\beta}\right)^{2}-n^{2} H^{4}-2 n \operatorname{tr} \phi_{h}^{2} .
$$

Proof. If $H=0$, we have $\phi_{\alpha}=-A_{\alpha}$ for all $\alpha$, and thus

$$
-\sum_{i, j, k, \ell, \alpha, \beta} h_{i j}^{\alpha} h_{k \ell}^{\alpha} h_{i j}^{\beta} h_{k \ell}^{\beta}=-\sum_{\alpha, \beta}\left(\operatorname{tr} A_{\alpha} A_{\beta}\right)^{2}=-\sum_{\alpha, \beta}\left(\operatorname{tr} \phi_{\alpha} \phi_{\beta}\right)^{2}
$$

which proves the lemma in this case. If $H \neq 0$, choose a local orthonormal frame $\left\{e_{n+1}, \ldots, e_{n+p}\right\}$ such that $e_{n+1}=h /|h|$. With this choice we have

$$
\left\{\begin{array}{l}
\phi_{n+1}=H I d-A_{n+1}, \\
\phi_{\alpha}=-A_{\alpha}, \alpha>n+1
\end{array}\right.
$$

and

$$
\left\{\begin{array}{l}
\operatorname{tr} A_{n+1}=n H, \\
\operatorname{tr} A_{\alpha}=0, \alpha>n+1 .
\end{array}\right.
$$

Furthermore, since $e_{n+1}$ is a parallel direction, we have $\left[A_{n+1}, A_{\beta}\right]=0$, and 
thus $\left[\phi_{n+1}, \phi_{\beta}\right]=0$ for all $\beta$. Using (3.20) and (3.21), we have

$$
\begin{aligned}
& -\sum_{i, j, k, \ell, \alpha, \beta} h_{i j}^{\alpha} h_{k \ell}^{\alpha} h_{i j}^{\beta} h_{k \ell}^{\beta}=-\sum_{\alpha, \beta}\left(\operatorname{tr} A_{\alpha} A_{\beta}\right)^{2} \\
= & -\sum_{\alpha, \beta>n+1}\left(\operatorname{tr} \phi_{\alpha} \phi_{\beta}\right)^{2}-2 \sum_{\alpha>n+1}\left(\operatorname{tr}\left(H I d-\phi_{n+1}\right) \phi_{\alpha}\right)^{2}-\left(\operatorname{tr}\left(H I d-\phi_{n+1}\right)^{2}\right)^{2} \\
= & -\sum_{\alpha, \beta>n+1}\left(\operatorname{tr} \phi_{\alpha} \phi_{\beta}\right)^{2}-2 \sum_{\alpha>n+1}\left(H \operatorname{tr} \phi_{\alpha}-\operatorname{tr} \phi_{n+1} \phi_{\alpha}\right)^{2} \\
& -\left(\operatorname{tr}\left(H^{2} I d-2 H \phi_{n+1}+\phi_{n+1}^{2}\right)\right)^{2} \\
= & -\sum_{\alpha, \beta>n+1}\left(\operatorname{tr} \phi_{\alpha} \phi_{\beta}\right)^{2}-2 \sum_{\alpha>n+1}\left(\operatorname{tr} \phi_{n+1} \phi_{\alpha}\right)^{2}-\left(n H^{2}+\operatorname{tr} \phi_{n+1}^{2}\right)^{2} \\
=- & \sum_{\alpha, \beta}\left(\operatorname{tr} \phi_{\alpha} \phi_{\beta}\right)^{2}-n^{2} H^{4}-2 n H^{2} \operatorname{tr} \phi_{n+1}^{2} \\
= & -\sum_{\alpha, \beta}\left(\operatorname{tr} \phi_{\alpha} \phi_{\beta}\right)^{2}-n^{2} H^{4}-2 n \operatorname{tr} \phi_{h}^{2},
\end{aligned}
$$

and the lemma is proved.

\section{Lemma 3.22.}

$$
\sum_{i, j, k, \ell, \alpha, \beta} h_{i j}^{\alpha} h_{i \ell}^{\alpha} h_{j \ell}^{\beta} h_{k k}^{\beta} \geq-\frac{n(n-2)}{\sqrt{n(n-1)}}\left|\phi_{h}\right||\phi|^{2}+2 n\left|\phi_{h}\right|^{2}+n H^{2}|\phi|^{2}+n^{2} H^{4} .
$$

Proof. Since the inequality is obvious if $H=0$, we can assume $H \neq 0$. As in the previous lemma, choose a local orthonormal frame $\left\{e_{n+1}, \ldots, e_{n+p}\right\}$ so that $e_{n+1}=h /|h|$. Then (3.20) and (3.21) hold, and we have

$$
\begin{aligned}
& \sum_{i, j, k, \ell, \alpha, \beta} h_{i j}^{\alpha} h_{i \ell}^{\alpha} h_{j \ell}^{\beta} h_{k k}^{\beta}=\sum_{\alpha, \beta}\left(\operatorname{tr} A_{\alpha}\right)\left(\operatorname{tr} A_{\alpha} A_{\beta}^{2}\right) \\
= & n H \sum_{\alpha} \operatorname{tr} A_{n+1} A_{\alpha}^{2} \\
= & n H \sum_{\alpha>n+1} \operatorname{tr}\left(H I d-\phi_{n+1}\right) \phi_{\alpha}^{2}+n H \operatorname{tr}\left(H I d-\phi_{n+1}\right)^{3} \\
= & n H^{2} \sum_{\alpha>n+1} \operatorname{tr} \phi_{\alpha}^{2}-n H \sum_{\alpha>n+1} \operatorname{tr} \phi_{n+1} \phi_{\alpha}^{2} \\
& +n H \operatorname{tr}\left(H^{3} I d-3 H^{2} \phi_{n+1}+3 H \phi_{n+1}^{2}-\phi_{n+1}^{3}\right) \\
= & n H^{2} \sum_{\alpha>n+1} \operatorname{tr} \phi_{\alpha}^{2}-n H \sum_{\alpha} \operatorname{tr} \phi_{n+1} \phi_{\alpha}^{2}+n^{2} H^{4}+3 n H^{2} \operatorname{tr} \phi_{n+1}^{2} \\
= & n H^{2}|\phi|^{2}-n H \sum_{\alpha} \operatorname{tr} \phi_{n+1} \phi_{\alpha}^{2}+n^{2} H^{4}+2 n H^{2} \operatorname{tr} \phi_{n+1}^{2} .
\end{aligned}
$$


Fix $\alpha$ and choose an orthonormal frame $\left\{e_{1}, \ldots, e_{n}\right\}$ such that $\phi_{i j}^{n+1}=$ $\mu_{i}^{n+1} \delta_{i j}$ and $\phi_{i j}^{\alpha}=\mu_{i}^{\alpha} \delta_{i j}$. This is possible since $\phi_{\alpha}$ and $\phi_{n+1}$ commute. Using Proposition (3.5) and the fact that $\operatorname{tr} \phi_{\alpha}=0$ for each $\alpha$, we have

$$
\begin{aligned}
\operatorname{tr} \phi_{n+1} \phi_{\alpha}^{2} & =\sum_{i}\left(\mu_{i}^{\alpha}\right)^{2} \mu_{i}^{n+1} \\
& \leq \frac{n-2}{\sqrt{n(n-1)}}\left(\sum_{i}\left(\mu_{i}^{\alpha}\right)^{2}\right)\left(\sum_{i}\left(\mu_{i}^{n+1}\right)^{2}\right)^{1 / 2} \\
& =\frac{n-2}{\sqrt{n(n-1)}}\left|\phi_{\alpha}\right|^{2}\left|\phi_{n+1}\right|
\end{aligned}
$$

and so

$$
\sum_{\alpha} \operatorname{tr} \phi_{n+1} \phi_{\alpha}^{2} \leq \frac{n-2}{\sqrt{n(n-1)}}\left|\phi_{n+1}\right||\phi|^{2}
$$

The lemma now follows from (3.23) and (3.24) if we observe that $\left|\phi_{h}\right|=$ $H\left|\phi_{n+1}\right|$ and $H^{2} \operatorname{tr} \phi_{n+1}^{2}=\operatorname{tr} \phi_{h}^{2}=\left|\phi_{h}\right|^{2}$.

We can finally prove Theorem (1.13).

Proof of Theorem (1.13). Integrating (3.2) and using Stokes's theorem, we obtain from Lemmas (3.12), (3.16), (3.19) and (3.22) that

$$
\begin{aligned}
0 \geq \int_{M}\left\{-E(b-a)^{2}+n a|\phi|^{2}\right. & -\frac{2}{3}(n-1)^{\frac{1}{2}}(p-1)(b-a)|A|^{2}-\frac{n(n-2)}{\sqrt{n(n-1)}}\left|\phi_{h}\right||\phi|^{2} \\
& \left.+n H^{2}|\phi|^{2}+\sum_{\alpha, \beta} \operatorname{tr}\left[A_{\alpha}, A_{\beta}\right]^{2}-\sum_{\alpha, \beta}\left(\operatorname{tr} \phi_{\alpha} \phi_{\beta}\right)^{2}\right\}
\end{aligned}
$$

since

$$
\sum_{\alpha, \beta} \operatorname{tr}\left[A_{\alpha}, A_{\beta}\right]^{2}=-\sum_{i, j, k, \ell, \alpha, \beta}\left(h_{i k}^{\alpha} h_{j k}^{\beta}-h_{j k}^{\alpha} h_{i k}^{\beta}\right)\left(h_{i \ell}^{\alpha} h_{j \ell}^{\beta}-h_{j \ell}^{\alpha} h_{i \ell}^{\beta}\right) .
$$

If $H=0$, we have $\phi_{\alpha}=-A_{\alpha}$ for all $\alpha$, and from Proposition (3.6) we obtain

$$
\begin{aligned}
\sum_{\alpha, \beta} \operatorname{tr}\left[A_{\alpha}, A_{\beta}\right]^{2}-\sum_{\alpha, \beta}\left(\operatorname{tr} \phi_{\alpha} \phi_{\beta}\right)^{2} & =-\sum_{\alpha, \beta} N\left(\phi_{\alpha} \phi_{\beta}-\phi_{\beta} \phi_{\alpha}\right)-\sum_{\alpha, \beta}\left(\operatorname{tr} \phi_{\alpha} \phi_{\beta}\right)^{2} \\
& \geq-\left(1+\frac{1}{2} \operatorname{sgn}(p-1)\right)\left(\sum_{\alpha} \operatorname{tr} \phi_{\alpha}^{2}\right)^{2} \\
& =-\left(1+\frac{1}{2} \operatorname{sgn}(p-1)\right)|\phi|^{4}
\end{aligned}
$$


If $p=1$, we clearly have

$$
\sum_{\alpha, \beta} \operatorname{tr}\left[A_{\alpha}, A_{\beta}\right]^{2}-\sum_{\alpha, \beta}\left(\operatorname{tr} \phi_{\alpha} \phi_{\beta}\right)^{2}=-|\phi|^{4} .
$$

Suppose now that $H \neq 0$ and $p \geq 2$. Choose $\left\{e_{n+1}, \ldots, e_{n+p}\right\}$ such that $e_{n+1}=h /|h|$. So $\left[A_{n+1}, A_{\beta}\right]=0$ for all $\beta,(3.20)$ holds and we obtain

$$
\begin{aligned}
\sum_{\alpha, \beta} \operatorname{tr}\left[A_{\alpha}, A_{\beta}\right]^{2} & -\sum_{\alpha, \beta}\left(\operatorname{tr} \phi_{\alpha} \phi_{\beta}\right)^{2}=-\sum_{\alpha, \beta>n+1} N\left(\phi_{\alpha} \phi_{\beta}-\phi_{\beta} \phi_{\alpha}\right) \\
& -\sum_{\alpha, \beta>n+1}\left(\operatorname{tr} \phi_{\alpha} \phi_{\beta}\right)^{2}-2 \sum_{\alpha>n+1}\left(\operatorname{tr} \phi_{n+1} \phi_{\alpha}\right)^{2}-\left|\phi_{n+1}\right|^{4} .
\end{aligned}
$$

Applying Proposition (3.6) for the matrices $\phi_{n+2}, \ldots, \phi_{n+p}$ we have

$$
\begin{aligned}
-\sum_{\alpha, \beta>n+1} N\left(\phi_{\alpha} \phi_{\beta}-\phi_{\beta} \phi_{\alpha}\right) & -\sum_{\alpha, \beta>n+1}\left(\operatorname{tr} \phi_{\alpha} \phi_{\beta}\right)^{2} \\
\geq & -\left(1+\frac{1}{2} \operatorname{sgn}(p-2)\right)\left(\sum_{\alpha>n+1} \operatorname{tr} \phi_{\alpha}^{2}\right)^{2} \\
= & -\left(1+\frac{1}{2} \operatorname{sgn}(p-2)\right)\left(|\phi|^{2}-\left|\phi_{n+1}\right|^{2}\right)^{2} .
\end{aligned}
$$

On the other hand, by the Cauchy-Schwarz inequality we obtain

$$
\left(\operatorname{tr} \phi_{n+1} \phi_{\alpha}\right)^{2} \geq\left(\operatorname{tr} \phi_{n+1}^{2}\right)\left(\operatorname{tr} \phi_{\alpha}^{2}\right)=\left|\phi_{n+1}\right|^{2}\left|\phi_{\alpha}\right|^{2},
$$

and thus

$$
-2 \sum_{\alpha>n+1}\left(\operatorname{tr} \phi_{n+1} \phi_{\alpha}\right)^{2} \geq-2\left|\phi_{n+1}\right|^{2}\left[|\phi|^{2}-\left|\phi_{n+1}\right|^{2}\right]
$$

By (3.26), (3.27) and (3.28), we have

$$
\begin{aligned}
\sum_{\alpha, \beta} \operatorname{tr}\left[A_{\alpha}, A_{\beta}\right]^{2}-\sum_{\alpha, \beta}\left(\operatorname{tr} \phi_{\alpha} \phi_{\beta}\right)^{2} \geq & -\left(1+\frac{1}{2} \operatorname{sgn}(p-2)\right)\left(|\phi|^{2}-\left|\phi_{n+1}\right|^{2}\right)^{2} \\
& -2\left|\phi_{n+1}\right|^{2}\left(|\phi|^{2}-\left|\phi_{n+1}\right|^{2}\right)-\left|\phi_{n+1}\right|^{4} \\
= & -\left(1+\frac{1}{2} \operatorname{sgn}(p-2)\right)|\phi|^{4} \\
& +\frac{1}{2} \operatorname{sgn}(p-2)\left|\phi_{n+1}\right|^{2}\left(2|\phi|^{2}-\left|\phi_{n+1}\right|^{2}\right) .
\end{aligned}
$$

Since

$$
\frac{1}{2} \operatorname{sgn}(p-2)\left|\phi_{n+1}\right|^{2}\left[2|\phi|^{2}-\left|\phi_{n+1}\right|^{2}\right] \geq 0,
$$


we conclude that

$$
\sum_{\alpha, \beta} \operatorname{tr}\left[A_{\alpha}, A_{\beta}\right]^{2}-\sum_{\alpha, \beta}\left(\operatorname{tr} \phi_{\alpha} \phi_{\beta}\right)^{2} \geq-\left(1+\frac{1}{2} \operatorname{sgn}(p-2)\right)|\phi|^{4} .
$$

Therefore, in any case we have

$$
\sum_{\alpha, \beta} \operatorname{tr}\left[A_{\alpha}, A_{\beta}\right]^{2}-\sum_{\alpha, \beta}\left(\operatorname{tr} \phi_{\alpha} \phi_{\beta}\right)^{2} \geq-\theta_{p, h}|\phi|^{4},
$$

where $\theta_{p, h}$ is given by (1.15). The conclusion of the theorem now follows from (1.9), (3.25) and (3.30).

\section{Proof of Theorems (1.23) and (1.32).}

In order to prove Theorem (1.23), we will need the following results:

Theorem 4.1 [10]. Let $M^{n}$ be a compact submanifold in $S_{1}^{n+p}$ with parallel mean curvature vector $h$ and $R^{\perp}=0$. If $\phi$ satisfies

$$
|\phi|^{2} \leq n\left(1+H^{2}\right)-\frac{n(n-2)}{\sqrt{n(n-1)}}\left|\phi_{h}\right|
$$

then

(i) $|\phi|$ is constant and either $|\phi|^{2} \equiv 0$ (and $M^{n}$ is totally umbilic) or the equality in (4.2) holds.

(ii) Equality holds in (4.2) if and only if:

(a) $M^{n}$ is a Clifford torus $S^{m}\left(r_{1}\right) \times S^{n-m}\left(r_{2}\right) \subset S_{1+H^{2}}^{n+1} \underset{u}{\longrightarrow} \longrightarrow S_{1}^{n+p}$, where $r_{1}=\left(\frac{m}{n\left(1+H^{2}\right)}\right)^{1 / 2}$ and $r_{2}=\left(\frac{n-m}{n\left(1+H^{2}\right)}\right)^{1 / 2}$.

(b) For every $H_{o}, 0 \leq H_{o}<H, M^{n}$ is an $H_{1}(r)$-torus $S^{n-1}(r) \times$ $S^{1}\left(r_{1}\right) \subset S_{1+H_{o}^{2}}^{n+1} \rightarrow \longrightarrow S_{1}^{n+p}$, where $H_{o}^{2}+H_{1}^{2}=H^{2}, r^{2}+r_{1}^{2}=$ $\left(1+H_{o}^{2}\right)^{-1}$. If $n \geq 3$, we have $r^{2}<\frac{n-1}{n}\left(1+H_{o}^{2}\right)^{-1}$, and if $n=2$, we have $r^{2} \neq \frac{1}{2}\left(1+H_{o}^{2}\right)^{-1}$.

A well-known theorem of Chern-do Carmo-Kobayashi [4] states that if $M^{n}$ is an oriented closed minimal submanifold in the unit sphere $S_{1}^{n+p}$ such that $|A|^{2} \leq n /(2-1 / p)$, then either $M$ is the unit sphere $S_{1}^{n}$, one of the Clifford minimal hypersurfaces in $S_{1}^{n+1}$, or the Veronese surface in $S_{1}^{4}$. For $p \geq 2$, A.M. Li and J.M. Li [8] obtained the Chern-do Carmo-Kobayashi's Theorem under the weaker assumption that $|A|^{2} \leq 2 n / 3$. More precisely, they proved the following theorem:

Theorem 4.3 [8]. Let $M^{n}$ be an $n$-dimensional compact minimal submanifold in $S_{1}^{n+p}, p \geq 2$. If $|A|^{2} \leq \frac{2 n}{3}$ everywhere on $M$, then $M$ is either a totally geodesic submanifold or a Veronese surface in $S_{1}^{4}$. 
An immersion $f: M^{n} \rightarrow N^{n+p}$ is pseudo-umbilic if the mean curvature vector $h \neq 0$ is an umbilical direction. The following theorem was proved by S.P. Chen ([3], see also [10]).

Theorem 4.4 [3]. Let $M^{n}$ be a compact pseudo-umbilical submanifold in $S_{1}^{n+p}, p \geq 2$, and suppose the mean curvature vector $h$ is parallel in the normal connection. If

$$
|\phi|^{2} \leq \frac{p-1}{2 p-3} n\left(1+H^{2}\right)
$$

then

(i) Either $|\phi|^{2} \equiv 0$ (and $M^{n}$ is totally umbilic) or the equality holds in (4.5).

(ii) Equality holds in (4.5) if and only if:

- (a) $p=2$ and $M^{n}$ is a Clifford torus $S^{m}\left(r_{1}\right) \times S^{n-m}\left(r_{2}\right) \subset$ $S_{1+H^{2}}^{n+1} \underset{u}{\longrightarrow} \longrightarrow S_{1}^{n+2}$, where $r_{1}=\left(\frac{m}{n\left(1+H^{2}\right)}\right)^{1 / 2}$ and $r_{2}=\left(\frac{n-m}{n\left(1+H^{2}\right)}\right)^{1 / 2}$.

- (b) $n=2, p=3$ and $M$ is the Veronese surface $M^{2} \subset S_{1+H^{2}}^{4} \vec{u}$ $\longrightarrow S_{1}^{5}$.

Remark 4.6. The proof of Theorem (4.4) gived by Santos [10] uses the theorem of Chern-do Carmo-Kobayashi [4]. If instead of this theorem one uses Theorem (4.3), the same proof shows that hypothesis (4.5) in Theorem (4.4) can be replaced by

$$
|\phi|^{2} \leq \frac{n\left(1+H^{2}\right)}{1+\frac{1}{2} \operatorname{sgn}(p-2)} .
$$

We can now prove Theorem (1.23):

Proof of Theorem (1.23). Since $c \leq K_{N} \leq 1$, we have $c \leq a(x) \leq b(x) \leq 1$, 
for every $x \in M$, and from (1.18) we obtain

$$
\begin{aligned}
& 0 \geq \int_{M}\left\{-E(b-a)^{2}-\frac{2}{3} n(n-1)^{\frac{1}{2}}(p-1)(b-a) H^{2}\right. \\
& +\left(n a+n H^{2}-\frac{2}{3}(n-1)^{\frac{1}{2}}(p-1)(b-a)\right)|\phi|^{2} \\
& \left.-\frac{n(n-2)}{\sqrt{n(n-1)}} H|\phi|^{3}-\theta_{p, h}|\phi|^{4}\right\} \\
& \geq \int_{M}\left\{-E(1-c)^{2}-\frac{2}{3} n(n-1)^{\frac{1}{2}}(p-1)(1-c) H^{2}\right. \\
& +\left(n c+n H^{2}-\frac{2}{3}(n-1)^{\frac{1}{2}}(p-1)(1-c)\right)|\phi|^{2} \\
& \left.-\frac{n(n-2)}{\sqrt{n(n-1)}} H|\phi|^{3}-\theta_{p, h}|\phi|^{4}\right\}=\int_{M} P_{H, c, p}(|\phi|),
\end{aligned}
$$

where $P_{H, c, p}(x)$ is the polynomial given by $(1.22)$.

Now we want to show that the polynomial $P_{H, c, p}(x)$ of fourth degree in $x$ has exactly two positive real roots. By observing the sign of the coefficients of $P_{H, c, p}(x)$, we conclude from the Descartes' rule of sign (see [9], page 60, Corollary 35) that $P_{H, c, p}(x)=0$ has at most two positive real roots $x_{1}(c) \leq x_{2}(c)$. Since $P_{H, c, p}(0) \leq 0$ and $P_{H, c, p}(x) \rightarrow-\infty$ as $x \rightarrow \infty$, it suffices to show that $P_{H, c, p}\left(x_{o}\right) \geq 0$ for some $x_{o}>0$. We claim that

$$
P_{H, c, p}\left(E^{\frac{1}{4}}(1-c)^{\frac{1}{2}}\right) \geq 0 .
$$

A simple computation shows that

$$
\begin{aligned}
& P_{H, c, p}\left(E^{\frac{1}{4}}(1-c)^{\frac{1}{2}}\right)=(1-c)\{-F(n, p, H)(1-c) \\
&\left.\quad-\frac{n(n-2)}{\sqrt{n(n-1)}} H E^{\frac{3}{4}}(1-c)^{\frac{1}{2}}+G(n, p, H)\right\} \\
&=(1-c) J(1-c),
\end{aligned}
$$

where $F(n, p, H)$ and $G(n, p, H)$ are given by (1.19) and (1.20), respectively, and

$$
J(1-c)=-F(n, p, H)(1-c)-\frac{n(n-2)}{\sqrt{n(n-1)}} H E^{\frac{3}{4}}(1-c)^{\frac{1}{2}}+G(n, p, H) .
$$

The hypothesis (1.24) implies that $\sqrt{1-c} \leq \lambda(n, p, H)$ (note that $\lambda(n, p, H)>$ 0 since $G(n, p, H)>0)$, and it is easy to see that $J(1-c) \geq 0$. The claim 
now follows from (4.9). We have, therefore, exactly two positive real roots $x_{1}(c) \leq x_{2}(c)$. Furthermore, we have $P_{H, c, p}(x) \geq 0$ for every $x \in\left[x_{1}, x_{2}\right]$.

Using condition (1.25), it then follows from (4.8) that

$$
0 \geq \int_{M} P_{H, c, p}(|\phi|) \geq 0
$$

and, therefore, $P_{H, c, p}(|\phi|) \equiv 0$. So, all the above inequalities become equalities. From the second equality in (4.8), we obtain

$$
\left\{\begin{array}{l}
b(x)=1, \\
a(x)=c .
\end{array}, x \in M\right.
$$

On the other hand, the fourth equality in (3.14) and the fourth equality in (3.18) give, respectively,

$$
\begin{gathered}
\left|K_{j i j}^{\alpha}\right|=\frac{1}{2}(b-a), \text { for all } \alpha \text { and } i \neq j \\
K_{\ell k \ell}^{k}=a, \text { for some pair }(k, \ell), k \neq \ell .
\end{gathered}
$$

Using (4.11), (4.12) and the equality case in Proposition (3.4)(i), we have

$$
K_{\alpha \ell}=K_{k \ell}=a, \quad \text { for all } \alpha,
$$

and from (3.7) we obtain $\left|K_{\ell k \ell}^{\alpha}\right|=0$, for all $\alpha$. It then follows from (4.10) and (4.11) that $c=1$. Since $N^{n+p}$ is complete and simply connected, we have $N^{n+p}=S_{1}^{n+p}$.

Condition (1.25) now becomes

$$
|\phi|^{2} \leq B_{H, p}
$$

where

$$
\sqrt{B_{H, p}}=\frac{-\frac{n(n-2)}{\sqrt{n(n-1)}} H+\sqrt{\frac{n(n-2)^{2}}{n-1} H^{2}+4 n\left(1+H^{2}\right) \theta_{p, h}}}{2 \theta_{p, h}},
$$

and from (4.8) we conclude that either $|\phi|^{2} \equiv 0$ (and $M^{n}$ is totally umbilic) or $|\phi|^{2} \equiv B_{H, p}$.

We now characterize all $M^{n}$ for which $|\phi|^{2} \equiv B_{H, p}$. If $p=1$, we have

$$
|\phi|=\frac{-\frac{n(n-2)}{\sqrt{n(n-1)}} H+\sqrt{\frac{n(n-2)^{2}}{n-1} H^{2}+4 n\left(1+H^{2}\right)}}{2}=\sqrt{B_{H}},
$$


and the conclusion follows from Theorem (1.12). If $H=0$ and $p \geq 2$, we have $|A|^{2}=|\phi|^{2}=2 n / 3$, and the conclusion follows from Theorem (4.3). If $p \geq 2$ and $H \neq 0$, we observe that equality in (1.17) implies that $n=2$ or $\left|\phi_{n+1}\right|=|\phi|$, while equality in (3.29) gives that $p=2$ or $\left|\phi_{n+1}\right|=0$.

We now investigate all possibilities. If $p=2$ and $n=2$, we have $R^{\perp}=0$ (since $h$ is a parallel direction) and

$$
|\phi|^{2}=2\left(1+H^{2}\right)
$$

and the conclusion follows from Theorem (4.1). If $p=2$ and $\left|\phi_{n+1}\right|=|\phi|$, we have $\left|A_{n+2}\right|=\left|\phi_{n+2}\right|=0$, which means that $N^{1} \subset \operatorname{span}\left\{e_{n+1}\right\}$, where

$$
N^{1}=\operatorname{span}\{\alpha(X, Y) ; X, Y \in T M\}
$$

is the first normal space. Since $e_{n+1}$ is a parallel direction, it follows from a Theorem of Dajczer [5] that the codimension can be reduced to $p=1$. Furthermore, equation (4.15) is satisfied and then the conclusion follows from Theorem (1.12). Finally, if $\left|\phi_{n+1}\right|=0$ and $n=2$, the immersion is pseudo-umbilic, and $|\phi|^{2} \equiv B_{H, p}$ becomes

$$
|\phi|^{2}=\frac{2\left(1+H^{2}\right)}{1+\frac{1}{2} \operatorname{sgn}(p-2)},
$$

and the conclusion follows from Theorem (4.4) (see Remark (4.6)). The proof of the Theorem is now complete.

Proof of Theorem (1.32). From hypothesis (1.31) we obtain

$$
c \geq 1-\frac{B_{H}}{n+2 E^{1 / 2}},
$$

which implies that

$$
E^{\frac{1}{2}}(1-c) \leq B_{H}-\left(n+E^{\frac{1}{2}}\right)(1-c) .
$$

On the other hand, a simple computation gives

$$
1-\lambda^{2}(n, 1, H) \leq 1-\frac{B_{H}}{n+2 E^{1 / 2}}
$$

and our assumption (1.31) implies that

$$
1-\lambda^{2}(n, 1, H) \leq K_{N} \leq 1 .
$$

If we show that

$$
P_{H, c, 1}\left(E^{\frac{1}{4}}(1-c)^{\frac{1}{2}}\right) \geq 0
$$


and

$$
P_{H, c, 1}\left(\sqrt{B_{H}-\left(n+E^{1 / 2}\right)(1-c)}\right) \geq 0,
$$

we conclude that $P_{H, c, 1}(x)=0$ has exactly two positive real roots $x_{1} \leq x_{2}$, and that

$$
x_{1} \leq E^{\frac{1}{4}}(1-c)^{\frac{1}{2}} \leq \sqrt{B_{H}-\left(n+E^{1 / 2}\right)(1-c)} \leq x_{2},
$$

and the conclusion of the theorem then follows from (1.33), (4.18), (4.21) and Theorem (1.23).

To complete the proof, it remains to prove (4.19) and (4.20). Inequality (4.19) follows from (4.18) and the proof of Theorem (1.23). To prove (4.20), we set $\beta=n+E^{1 / 2}$ and we have

$$
\begin{aligned}
& P_{H, c, 1}\left(\sqrt{B_{H}-\beta(1-c)}\right)=\left(B_{H}-\beta(1-c)\right)\left\{-\left(B_{H}-\beta(1-c)\right)\right. \\
& \left.\quad-\frac{n(n-2)}{\sqrt{n(n-1)}} H \sqrt{B_{H}-\beta(1-c)}+n\left(H^{2}+1\right)\right\} \\
& \quad-n\left(B_{H}-\beta(1-c)\right)+n c\left(B_{H}-\beta(1-c)\right)-E(1-c)^{2} \\
& \geq \\
& \quad\left(B_{H}-\beta(1-c)\right)\left\{-B_{H}-\frac{n(n-2)}{\sqrt{n(n-1)}} H B_{H}^{1 / 2}+n\left(H^{2}+1\right)\right\} \\
& +\left(B_{H}-\beta(1-c)\right) \beta(1-c)-n\left(B_{H}-\beta(1-c)\right)+n c\left(B_{H}-\beta(1-c)\right) \\
& -E(1-c)^{2} .
\end{aligned}
$$

Since $\sqrt{B_{H}}$ is the positive root of the polynomial $P_{H}(x)$ defined by (1.11), it follows from (4.17) that

$$
\begin{aligned}
P_{H, c, 1}\left(\sqrt{B_{H}-\beta(1-c)}\right) & \geq\left(B_{H}-\beta(1-c)\right)[\beta(1-c)-n+n c]-E(1-c)^{2} \\
& =\left(B_{H}-\beta(1-c)\right) E^{1 / 2}(1-c)-E(1-c)^{2} \\
& \geq 0 .
\end{aligned}
$$

The proof of the theorem is now complete.

\section{References}

[1] H. Alencar and M. do Carmo, Hypersurfaces with constant mean curvature in spheres, Proc. Amer. Math. Soc., 120 (1994), 1223-1229.

[2] H. Alencar and M. do Carmo, Hypersurfaces with constant mean curvature in space forms, Preprint. 
[3] S.P. Chen, Pseudo-umbilical submanifolds in spaces of constant curvature (In ChineseEnglish Summary), J. East China Norm Univ. Natur. Sci., 1 (1989), 59-65.

[4] S.S. Chern, M. do Carmo and S. Kobayashi, Minimal submanifold of a sphere with second fundamental form of constant length. In Functional Analysis and related fields, edited by F. Brower, Springer-Verlag, Berlin, 1970, 59-75.

[5] M. Dajczer, Reduction of codimension of regular isometric immersion, Math. Z., 179 (1982), 263-286.

[6] J. Erbacher, Isometric immersions of constant mean curvature and triviality of the norm connection, Nagoya Math. J., 45 (1971), 139-165.

[7] S.I. Goldberg, Curvature and homology, London Acad. Press Inc., 1962.

[8] A.M. Li and J.M. Li, An intrinsic rigidity theorem for minimal submanifolds in a sphere, Arch. Math., 58 (1992), 582-594.

[9] C.C. Mac Duffee, Theory of equations, John Wiley \& Sons Inc., 1954.

[10] W. Santos, Submanifolds with parallel mean curvature vector in spheres, Tôhoku Math. J., 46 (1994), 121-133.

[11] H. Xu, On closed minimal submanifolds in pinched riemannian manifolds, Preprint.

Received March 22, 1995.

Universidade Federal Fluminense

Instituto DE MATEMÁticA

Departamento de Geometria

Niterói - RJ

24.020-005 - BRASIL

E-mail address: Fonte@impa.br 